\title{
From gamma-ray bursts to fast radio bursts
}

\author{
S. R. Kulkarni \\ California Institute of Technology, Pasadena, CA 91125, USA
}

Gamma-ray bursts (GRBs) were discovered in late sixties by a team based at the Los Alamos National Laboratory but reported ${ }^{1}$ in June, 1973. Fast radio bursts (FRBs) were reported about a decade ago. ${ }^{2}$ GRBs and FRBs share two letters in their acronym, one common word and perhaps a similar history. Here, I review the development of the GRB field and use this occasion to apply lessons learnt during the early days of GRB to the rapidly developing field of FRBs.

Gamma-ray ( $\gamma$-ray) detectors have a large field-of-view but lack the ability to make images. The lack of localization meant that the distance to the bursts was unconstrained which, in turn, led to a frenzy of speculation. At the Seventh Texas Symposium (Dallas, December 1974) the Los Alamos team reported a total of 33 GRBs. Malvin Ruderman ${ }^{3}$ summarized the constraints posed by the observations to theories of GRBs and also listed the many more proposed models. The prevailing view was that GRBs were nearby old Galactic neutron stars.

The proceedings ${ }^{4}$ of a conference held at Taos (July, 1990) at the eve of the launch of the Compton Gamma-ray Observatory (CGRO) make for an excellent reading. The neutron star model rested on the presence of absorption lines in some GRBs. These were interpreted as cyclotron lines arising in the photosphere of neutron stars. The isotropic distribution meant that the neutron stars were nearby, distance $\approx 100 \mathrm{pc}$. The resulting energetics were modest. Triangulation of bright events, based on the arrival time provided by the Inter-Planetary Network (IPN) of satellites, led to localizations of a few square arc-minutes. The absence of optical quiescent counterparts was consistent with a neutron star origin. In any case, the Burst and Transient Source Experiment (BATSE) aboard $C G R O$ was expected to make definitive measurements of the sky distribution of faint events.

To be fair, suggestions for extra-galactic models were made in the eighties. In an influential paper ${ }^{5}$, Bohdan Paczyński, noting the isotropic distribution and the slow rise in the counts of fainter sources, advocated an extra-galactic model. He noted that the inferred isotropic $\gamma$-ray energy release was $10^{51} \mathrm{erg}$ - similar to the mechanical energy yield of supernovae. Paczyński strengthened his case by attributing three repeating bursts to an extragalactic GRB lensed by an intervening galaxy. However, no physical mechanism which could produce short bursts of $\gamma$-rays with high efficiency was identified.

By the time of the 1995 seminal review ${ }^{6}$ by Gerald Fishman and Charles Meegan, two classes had emerged: short duration with hard spectrum (SHB) and long duration but with soft spectrum (LSB). A subset, the eponymously named soft $\gamma$-ray repeaters (SGR) 
were accepted to be of Galactic origin (with the prototype, "5 March 1979" residing in the Large Magellanic Cloud). The three bursts discussed above had been identified with SGR 1900+14. Next, despite the increased sensitivity of BATSE, no absorption features were seen. Finally, the faint bursts were isotropically distributed which, in the Galactic framework, required the neutron stars to be located in the Galactic "corona" (distance $\approx 100 \mathrm{kpc}$ ). The introductory statement - "... adopt a new paradigm for the origin of $\gamma$-ray bursts: sources at cosmological distances. This new paradigm is almost entirely by default" - and the closing statement of the review - "The $\gamma$-ray enigma appears to be as great now as it was 20 years ago" - summarizes the mood of that era.

Another highlight of 1995 was the "The Great Debate" featuring Paczyński arguing for a cosmological origin and Donald Lamb for a Galactic origin. See https://apod. nasa.gov/debate/debate95.html for the program, presentations and photographs. I was present at this debate. Upon entry to the auditorium, attendees, based on their conviction, were expected to pick and wear one of three buttons: "Galactic", "Extragalactic" and "Other". I collected 'em all! There was no winner. The Galactic model was strained, requiring a goodly fraction of high velocity neutron stars with tailor-made bursting duty cycle. The extra-galactic model failed to explain the absorption lines. Both speakers advocated for a mission to search for GRBs towards our neighboring galaxy (M31).

Theoretical studies of extra-galactic origins accelerated. In an influential conference summary, Stanford Woosley ${ }^{7}$ discussed neutron star quakes, neutron phase transitions and the "collapsar" model (in which a natal black hole accretes matter copiously). Many focused on neutron star coalescences ${ }^{8}$ while others investigated long-lived radiation from

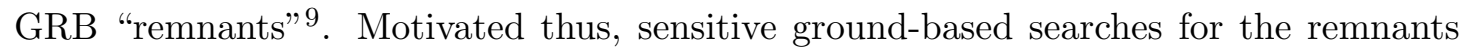
accelerated. Shortly thereafter, BeppoSAX discovered ${ }^{10}$ decaying X-ray emission from GRB 970228. The "afterglow" phenomenon - long lived (hours to months) emission at lower energies (X-rays through radio) - enabled arcsecond localization. This quickly led to a demonstration of the extra-galactic, nay, cosmological $(z \approx 0.8)$, origin $^{11}$ of LSBs. The spectroscopic observations of host galaxies linked LSBs preferentially to star-forming (albeit, dwarf) galaxies which suggested a massive star origin. The twelfth BeppoSAX event, GRB 980425, was very close, $45 \mathrm{Mpc}$, and became the prototype for the class of low luminosity GRBs. The event was rapidly identified with a mildly relativistic supernova - two firsts - and led to the induction of the word collapsar into the astronomical lexicon. Over time, "red bumps" were seen in optical afterglows of some "classical" LSBs - pointing to underlying supernovae. Pan-chromatic afterglows provided additional diagnostics: calorimetry, collimation angle of the relativistic ejecta, circumstellar medium density and angular size. Spectral features in X-ray afterglows were reported but also, as in the past, did not stand subsequent scrutiny and statistics.

The period May-July of 2005 was magical. Swift found the first X-ray afterglow of an SHB. Thereafter, HETE-2 and Swift successively localized two SHBs. Radio and optical afterglows were discovered. However, unlike LSBs, there were no associated bright supernovae. For one event, the host was a star-forming galaxy but an elliptical for the other! Swift then went on to accumulate samples of SHB host galaxies (including 
offsets) and followup observations for one SHB resulted in a tentative identification of a long-lived kilonova. Over time, the sub-classes of ultra-long duration GRBs and events with the bulk of their energy in the soft X-ray band ("X-ray flashes") have emerged. Searches for "dirty fireballs" (with peak energy in the ultra-violet or UV band) are continuing. Nonetheless, the declining production of papers shows the maturation of the GRB field (Figure 1).

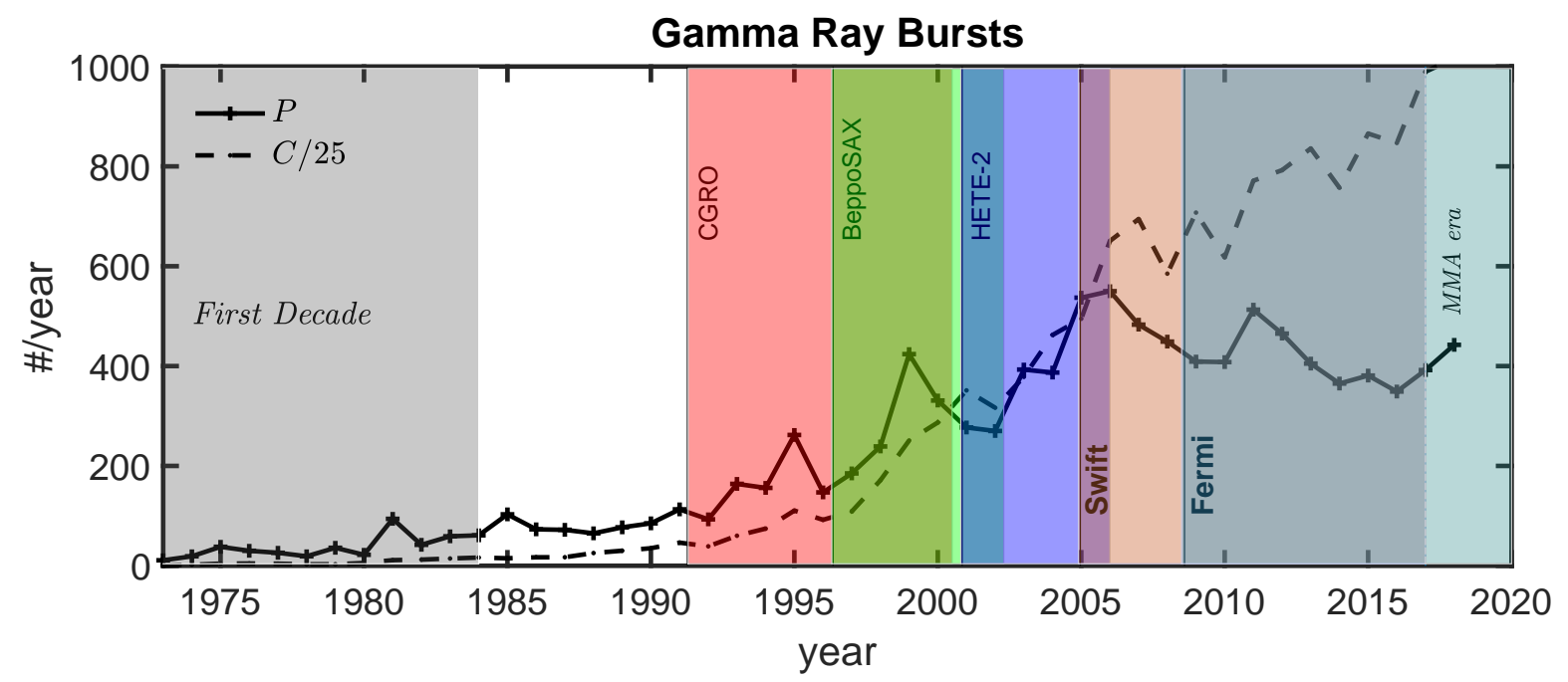

Figure 1: The annual flux of refereed papers $(P)$ and earned citations ( $C$, but divided by 25) of papers on or about GRBs since their discovery in 1973. Recognizing that there are 4 more months left in 2018, $P$ and $C$ have been boosted for this year by 3/2. The annual flux of papers is the number of papers published in a given year. In contrast, citations can be sorted by the year in which the paper was cited (earned) or tagged to the year in which the paper was published (accrued). Accrued citations, although commonly used, are not useful since they lag paper production by (typically) five years. To obtain the bibliographic data I used the "modern form" of Astrophysical Data System (ADS; http://adsabs.harvard.edu/) with the filter set to "GRB" or "gamma ray burst" (also $\gamma$-ray burst) in the title or abstract but with "solar", "blazar" and "Erratum" excluded. Over decades, Kevin Hurley of the IPN painstakingly assembled a list of GRB papers (http://www.ssl.berkeley.edu/ipn3/bibliogr.html). Hurley's list stands at 13,209 but includes conference proceedings and reports. My list is strictly limited to refereed papers and, as of September 2018, it stands at 10,374 papers with 388,036 citations. The left-most shaded region corresponds to the first decade and the right most to the burgeoning "Multi-Messenger Astronomy" (MMA) era. During the first decade the Soviet Venera missions made key contributions (isotropical distribution and the discovery of soft gamma-ray repeaters). The IPN was key to arcminute localization. Thin vertical lines mark the launch of key GRB missions (which are noted to the right of each line). CGRO, launched in April 1991, was the second of the four elements of the Great Observatories program. BeppoSAX, an Italian-Dutch mission, stands for Satellite per Astronomia a raggi $X$ $(S A X)$ and was named in honor of Giuseppe "Beppo" Occhialini who was the co-discoverer of the pion. The High Energy Transient Explorer-2 (HETE-2) was a small mission led out of the Massachusetts Institute of Technology. These three missions were terminated or ceased to operate in 2000, 2002 and 2006, respectively. The Neil Gehrels Swift Observatory, a "medium" explorer devoted to studies of GRBs, was launched in November, 2004. The Fermi $\gamma$-ray Space Telescope is a major NASA-DOE project aimed at studying the $\gamma$-ray sky. Both Swift and Fermi are still in operation.

It is time to review the lessons learnt from the development of GRB astronomy. The first lesson is, excluding solar bursts, that there are at least four major GRB phenomena: terrestrial gamma-ray flashes (from lightning in our atmosphere); SGRs, flares from strongly magnetized neutron stars, readily detected in our Galaxy; SHBs, coalescence 
of neutron stars, with typical redshift, $z \approx 0.8$; LSBs, deaths of a certain select type of massive stars, and can be seen to the edge of the Universe. The Galactic model was hobbled by fruitlessly trying to unify SGRs and GRBs.
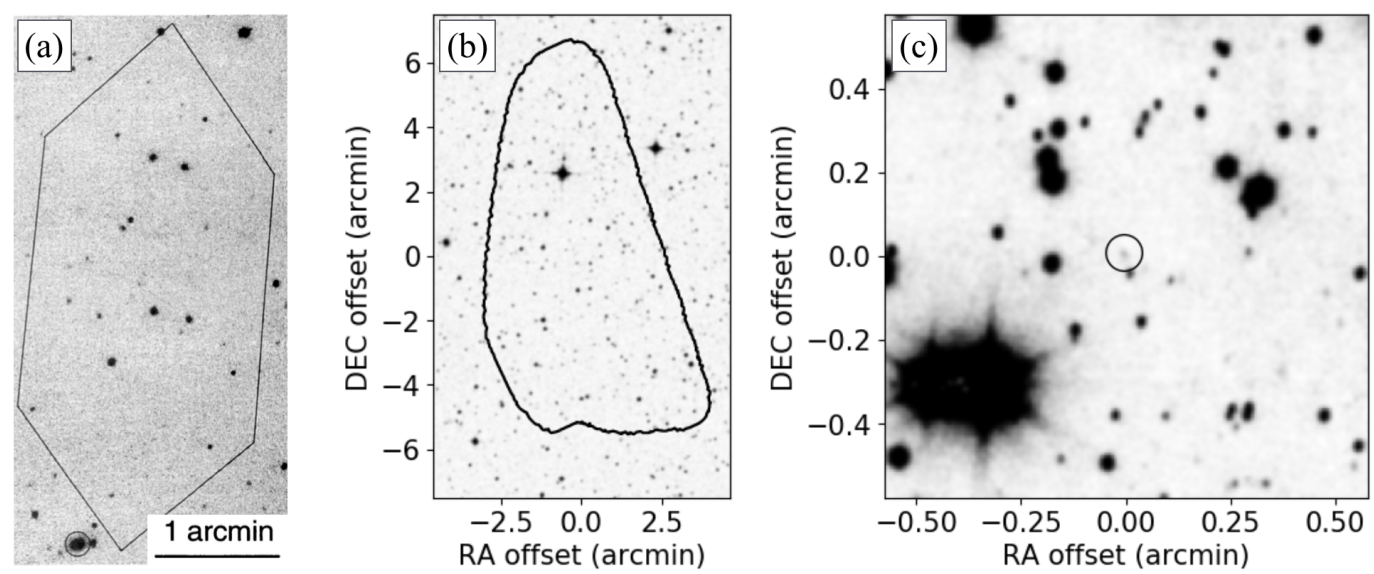

Figure 2: A selection of GRB and FRB localization regions. Panel (a) shows the IPN $99 \%$ confidence error box of GRB 910219 plotted on a deep Keck/NIRC K-band near-IR image. Panel (b) shows the 99\% localization region of the "Lorimer" burst (FRB 010724; ref. 2) obtained by comparing its detections in multiple beams of the Parkes multi-beam receiver. The background image is a composite of Second Digitized Sky Survey red and blue plates. Panel (c) shows a deep Keck/LRIS R-band image of the field of the Arecibo Repeater (FRB 121102; ref. 13). The Repeater has been localized by the Jansky Very Large Array to a dwarf galaxy (marked by a circle) remarkable only for its strong emission lines. Thanks to the astounding precision of Very Long Baseline Interferometry, it has been found that FRB 121102 is, to within milli-arcseconds, coincident with a strong non-thermal source which has been suggested to be an active galactic nucleus or a plerion. Credit: panel a reproduced from ref. 14, AAS.

Spectral lines top my list of red herrings. When two harmonically related absorption lines are reported it is difficult not to think of cyclotron lines and thence neutron star surfaces. Next, early IPN error boxes (dominated by LSBs) did not show bright galaxies. The huge range of galaxy luminosities compounded with the equally broad $\gamma$-ray luminosity function and the rapid redshift evolution of dwarf star-forming galaxies greatly weakened this clue (see Figure 2). Finally, relativistic beaming results in underestimating the distance (for a fixed isotropic energy) and hence over-estimating the host galaxy brightness.

It is said that it is tough to make predictions, especially about the future. However, the past, objectively analyzed, may improve our prediction of the future. The key lesson from GRBs is that the lack of a distance scale led to decades of speculations with little of lasting value. The great revolution in GRB astronomy can be directly linked to localization. The first facility which can routinely localize "classical" FRBs will become the BeppoSAX of the FRB field! Next, some clues carry more weight than others. The isotropy of faint events should have led to an immediate abandonment of the Galactic model. Finally, exceptional events (usually the nearest), if caught, are usually pivotal. HETE-2, a "low-cost explorer", was up when GRB 030329, one of the closest LSB exploded and thereby definitively connected LSBs to supernovae.

From Figure 3 we see that the FRB field, measured by citations, has moved at more 

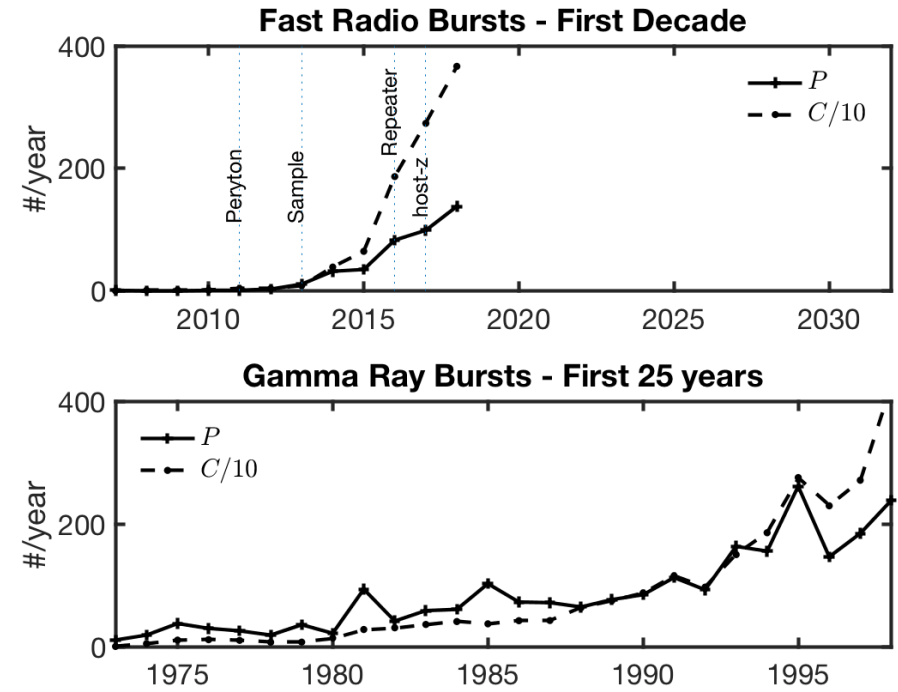

Figure 3: The annual flux of refereed papers, $P$ and citations, $C$ (but divided by 10) of FRB papers (top) and GRB papers (bottom). As in Figure 1 C and $P$ for FRB papers in 2018 were increased by 3/2. I followed a similar routine described in Figure 1 ADS was queried for papers with FRB or "fast radio burst" in the title or abstract. I then reviewed each entry and culled unrelated papers. Conference papers were ruthlessly excluded. Given the youth of this field, papers that were submitted but not yet published were included. Over the period 2007-September 2018 the FRB field produced 358 papers and racked up 8224 citations. Over a similar interval the GRB field had 396 papers but only 1630 citations. Clearly the FRB field has a higher tempo than that of GRBs. It took two decades for the GRB field to reach the citations that the FRB field achieved in its first decade. The contraction of timescales can be reasonably attributed to a larger pool of astronomers. For instance, membership of the American Astronomical Society increased from 3500 in 1980 to 7600 this year. The vertical lines mark key developments. "Perytons" shared some features with FRBs and cast grave doubts about the extraterrestrial nature of FRBs. Perytons were eventually localized to the Visitor's Centre of the Parkes Observatory. In retrospect Perytons are the equivalent of (photographic) "plate defects" (or, if we are lucky, the equivalent of spectral lines) of the GRB era. The FRB field really took off after the publication of a sample of four FRBs. The localization of the Arecibo Repeater and the subsequent identification of its host galaxy at $z \approx 0.19$ further increased the tempo of the field. Future progress will primarily be governed bythe rate of localizations. Over this and coming years several localization facilities are expected to come on line: ASKAP, TRAPUM/MeerKAT, UTMOST2D and DSA-110. 
than twice the speed of the GRB field. As an example, I note that two years ago a decaying radio source was linked to an FRB. This claim was investigated ferociously and persuasive arguments against were published within a month! This occasion made me wonder where the equivalent of spectral lines of the GRB era are lurking in the FRB field.

Based on the history of GRB light curves and radio pulsars, meaningful insights into the nature of FRB sources (let alone the emission mechanisms) are unlikely to come from details of the light curves. On the other hand, clues that arise from simpler phenomena - free-free absorption, rotation measures and multi-path propagation (scattering, scintillation) - will likely be powerful. CHIME with its large collecting area is superbly suited for this purpose.

With no doubt most of the FRBs are not in our Galaxy. Next, there are now two classes: (the so far sole) Arecibo Repeater and "classic" FRBs. The Repeater has been localized to a $z=0.19$ galaxy (Figure 2). The nature of host galaxies identified in the first phase of localization - star-forming galaxy, elliptical galaxy, intergalactic medium - will provide robust clues to the origin of FRBs. It will, however, require arc-second localization (see Figure 2). The offset of the events with respect to the galaxy components - nuclear, disk and halo- constitute the next phase but will need sub-arcsecond localization. Fortunately, an armada of radio localization facilities will be coming on line soon (Figure 3). FRB leaders, informed by the history of GRBs and Gravitational Wave astronomy, should take note of the vital importance of prompt dissemination of localization. Separately, inspired by SGRs, it would be worthwhile to look for Galactic versions of FRBs.

In preparing for this article I experienced deja $v u$ all over again. The modus operandi was astonishingly familiar: suggest all possible collisions between comets, asteroids, brown dwarfs, white dwarfs, neutron stars and black holes, invoke improbable scenarios (white dwarf and intermediate mass black hole in an eccentric orbit) and failing all these possibilities invoke string theory. The expected diversity of FRB classes should not be used as a license to speculate recklessly. A worthwhile model does not violate basic physical principles, identifies a mechanism which produces the observed signal strength and has an observationally meaningful rate. FRBs opened our eyes to extra-galactic millisecond bursts. In this spirt, searches for Fast Optical Bursts should be undertaken. Searches commensal with FRB surveys enjoy natural immunity against local errors (cf. the "plate defects" in the GRB era). I note that modern optical time domain surveys are finding ${ }^{12}$ afterglows even without GRB triggers!

The greatest impact of GRBs has been in stellar astronomy. LSBs have shown that some massive stars die with a hyper-relativistic explosion. Next, just over a year ago, Fermi discovered and promptly reported a faint short hard $\gamma$-ray burst. The burst was delayed two seconds relative to the merger of two neutron stars, the celebrated gravitational wave source GW 170817. This combination inaugurated the MMA era. A mission in a high orbit, perhaps named "Gamma ray Ultr $A$ Violet Astronomy SATellite (GUAVASAT!) carrying omni-directional $\gamma$-ray detectors tuned to SHBs and a UV widefield imager would be a strategic space-based mission for this era. GUAVASAT would 
also lift the declining fortunes of the GRB field (Figure 1).

The amazing diagnostics of FRBs - electron column density, magnetic field and turbulence - will most assuredly advance our understanding of the inter- and circumgalactic medium and perhaps even contribute to cosmography. Should it turn out that a class of FRBs is related to other phenomena (e.g. coalescence of neutron stars) or is truly of exotic origin (e.g. cosmic strings) then the FRB field will continue to flourish well into its third decade.

I thank Edwin Henneken, ADS/Center for Astrophysics, for help with ADS programming and Vikram Ravi for providing Figure 2, I gratefully acknowledge useful and enlightening discussions with M. Bailes, S. B. Cenko, D. A. Frail, D. A. Perley, E. S. Phinney \& V. Ravi. I thank K. Plant for careful reading and Caltech librarian J. Painter for help with in finding old references.

\section{REFERENCES}

1. Klebesadel, R. W., Strong, I. B. and Olson, R. A. Observations of Gamma-Ray Bursts of Cosmic Origin. Astrophys. J. 182, L85-L88 (1973).

2. Lorimer, D. R., Bailes, M., McLaughlin, M. A. et al. A Bright Millisecond Radio Burst of Extragalactic Origin. Science 318, 777-780 (2007).

3. Ruderman, M. Theories of $\gamma$-ray bursts. Annals NY Academy of Sciences 262, 164-180 (1975).

4. Ho, C., Epstein, R. I. and Fenimore, E. E. Gamma-ray bursts. Observations, analyses \& theories, Cambridge University Press, 516p. (1992)

5. Paczyński, B. Gamma-ray bursters at cosmological distances. Astrophys. J. 308, L43-L46 (1986)

6. Fishman, G. \& Meegan, C. Gamma-Ray Bursts. Annu. Rev. Astron. Astrophys. 33, 415-458 (1995)

7. Woosley, S. E. Theory of gamma-ray bursts. American Inst. Conf. Series 384, 709-718 (1997).

8. Piran, T. Gamma-ray Bursts and Binary Neutron Star Mergers. I. A. U. Symposium 165, 489-502 (1996).

9. Mészáros, P. \& Rees, M. J. Optical and Long-Wavelength Afterglow from Gamma-Ray Bursts. Astrophys. J. 476, 232-237 (1997).

10. Costa E., Frontera, F., Heise, J., Feroci, M., in't Zand, J. et al. Discovery of an X-ray afterglow associated with the $\gamma$-ray burst of 28 February 1997. Nature 387, 783-785 (1997).

11. Metzger, M. R., Djorgovski, S. G., Kulkarni, S. R., Steidel, C. C., Adelberger, K. L. et al. Spectral constraints on the redshift of the optical counterpart to the $\gamma$-ray burst of 8 May 1997. Nature 387, 878-880 (1997)

12. Cenko, S. B., Urban, A. L, Perley, D. A. et al. iPTF14yb: The First Discovery of a Gamma-Ray Burst Afterglow Independent of a High-energy Trigger. Astrophys. J. 803, L24-L28 (2015).

13. Spitler, L. G. Scholz, P., Hessels, J. W. T. et al. A repeating fast radio burst. Nature 531, 202-205 (2016)

14. Larson, S. B., McLean, I. S., Becklin, E. E. Luminous Galaxies near Gamma-Ray Burst Positions. Astrophys.

J. 460, L95-L97 (1996) 